\title{
Thermal characteristics of a B8.3 flare observed on July 04, 2009
}

\author{
Arun Kumar Awasthi ${ }^{1}$, Barbara Sylwester ${ }^{2}$, Janusz Sylwester ${ }^{2}$ and \\ Rajmal Jain ${ }^{3}$ \\ ${ }^{1}$ Astronomical Institute, University of Wroclaw, Poland \\ email: arun.awasthi.87@gmail.com \\ ${ }^{2}$ Space Research Center of Polish Academy of Sciences, Wroclaw, Poland \\ email: bs@cbk.pan.wroc.pl, js@cbk.pan.wroc.pl \\ ${ }^{3}$ Kadi Sarva Vishwavidyalaya, Gandhinagar, India \\ email: rajmal_9@yahoo.com
}

\begin{abstract}
We explore the temporal evolution of flare plasma parameters including temperature $(T)$ - differential emission measure $(D E M)$ relationship by analyzing high spectral and temporal cadence of X-ray emission in 1.6-8.0 keV energy band, recorded by SphinX (Polish) and Solar X-ray Spectrometer (SOXS; Indian) instruments, during a B8.3 flare which occurred on July 04, 2009. SphinX records X-ray emission in 1.2-15.0 keV energy band with the temporal and spectral cadence as good as $6 \mu \mathrm{s}$ and $0.4 \mathrm{keV}$, respectively. On the other hand, SOXS provides X-ray observations in 4-25 keV energy band with the temporal and spectral resolution of $3 \mathrm{~s}$ and $0.7 \mathrm{keV}$, respectively. We derive the thermal plasma parameters during impulsive phase of the flare employing well-established Withbroe-Sylwester DEM inversion algorithm.
\end{abstract}

Keywords. Sun: corona, Sun: flares, plasmas, Sun: X-rays, radiation mechanisms: thermal, techniques: spectroscopic.

\section{Introduction}

Thermal characteristics of solar flare plasma employing the multi-wavelength observations is of immense interest as it can shed light on the ongoing coupling processes in solar atmosphere. In particular, X-ray emission during a flare is the best probe of various thermal and non-thermal energy release processes (Brown (1971)). Generally, flare plasma parameters viz. temperature $(T)$, emission measure $(E M)$, etc. are derived by forward-fitting/inversion of the observed X-ray spectrum (Jain et al. (2011)). However, the spectroscopic inversion of X-ray emission is an ill-posed problem, leading to substantial uncertainties in the derived $T$ and $E M$ values (Craig \& Brown (1976)). Moreover, several different $D E M$ inversion techniques, with various functional dependence of $D E M$ on $T$ viz. power-law, single-gaussian etc., are used to interpret observed X-ray spectrum. Further, Withbroe-Sylwester (W-S) DEM inversion algorithm (Sylwester, Schrijver, \& Mewe (1980), Kepa et al. (2008)) provides a more general scheme for such studies.

Therefore, in this paper, we present the analysis of X-ray emission observed during a B8.3 flare occurred on July 04, 2009, the only event recorded in common with Solar X-ray Spectrometer (SOXS; Jain et al. (2005)) and Solar Photometer in X-rays (SphinX; Gburek et al. (2013)). Section 2 presents the observations while data analysis and results are given in Section 3. Section 4 presents the summary and conclusions. 

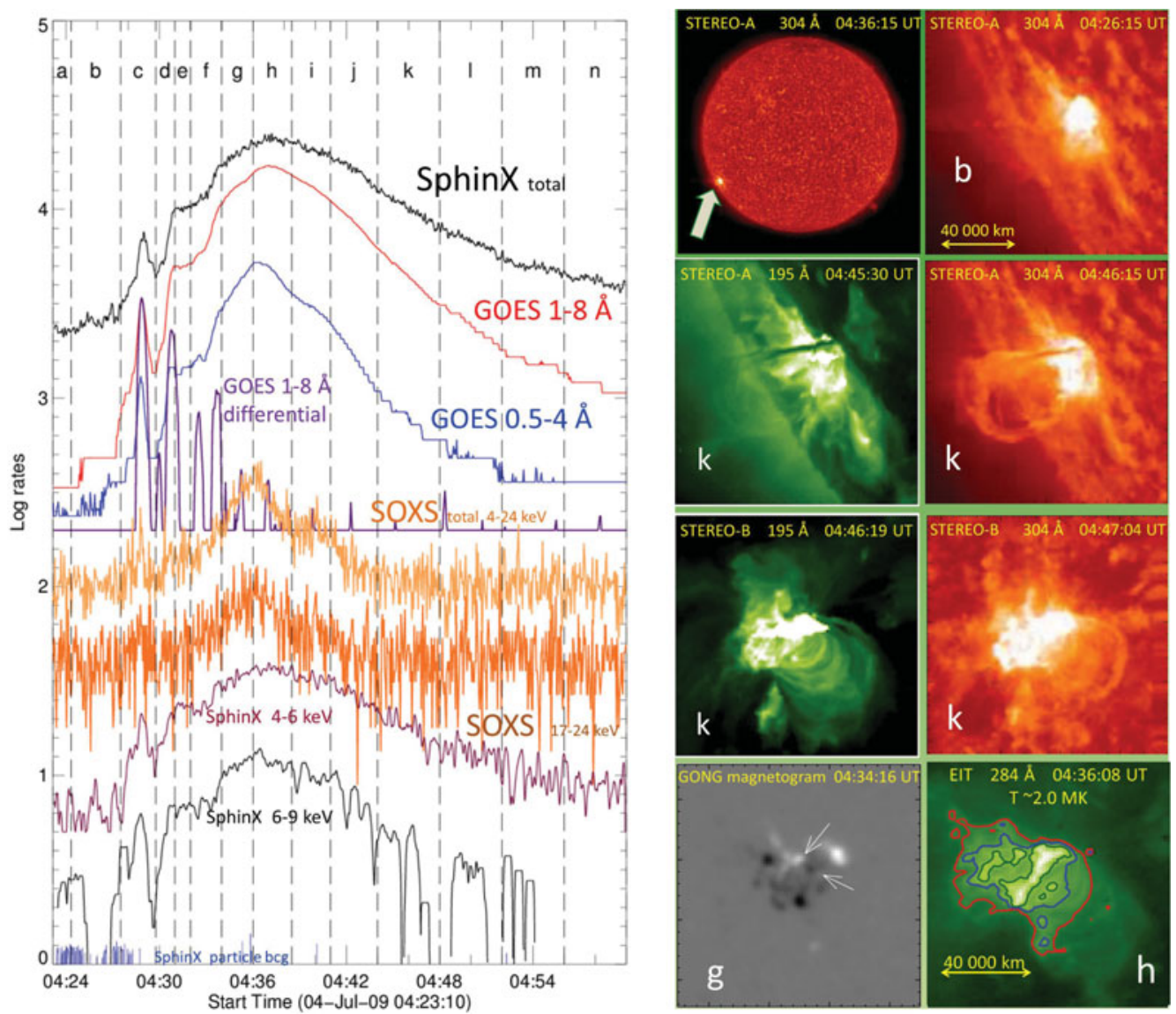

Figure 1. Left Panel: Temporal evolution of X-ray emission as recorded by SphinX, SOXS and GOES during SOL2009-07-04T04:37 (B8.3) flare. Dotted bars show the time intervals for which spectral analysis is undertaken. Right Panel: Multi-wavelength overview of the flare from STEREO-A and STEREO-B and EIT/SOHO. Activity areas are shown by arrows in the GONG Magnetogram (bottom).

\section{Observations}

We study a B8.3 flare event of July 04, 2009, which occurred in active region 11024 . Thermal characteristics of the flare plasma are derived by analyzing X-ray spectra in 1.6-5.0 keV and 5.0-8.0 keV energy bands, recorded by SphinX and SOXS, respectively. Temporal evolution of X-ray emission during the flare as observed by SphinX and SOXS instruments as well as by GOES is shown in the left panel of the Fig. 1. Further, morphological evolution of the flaring region is studied from the EUV images obtained from STEREO-A, B and Extreme Ultraviolet Imager Telescope (EIT) onboard SOHO mission, as shown in the right panel of the Fig. 1.

\section{Thermal characteristics of the flare plasma}

We analyze the X-ray spectra, recorded during the flare, with the help of W-S DEM inversion method (Sylwester, Schrijver, \& Mewe (1980)). This numerical method employs maximum likelihood approach in which a $D E M-T$ distribution and hence corresponding theoretical spectrum is derived in an iterative manner with the aim to minimize its 

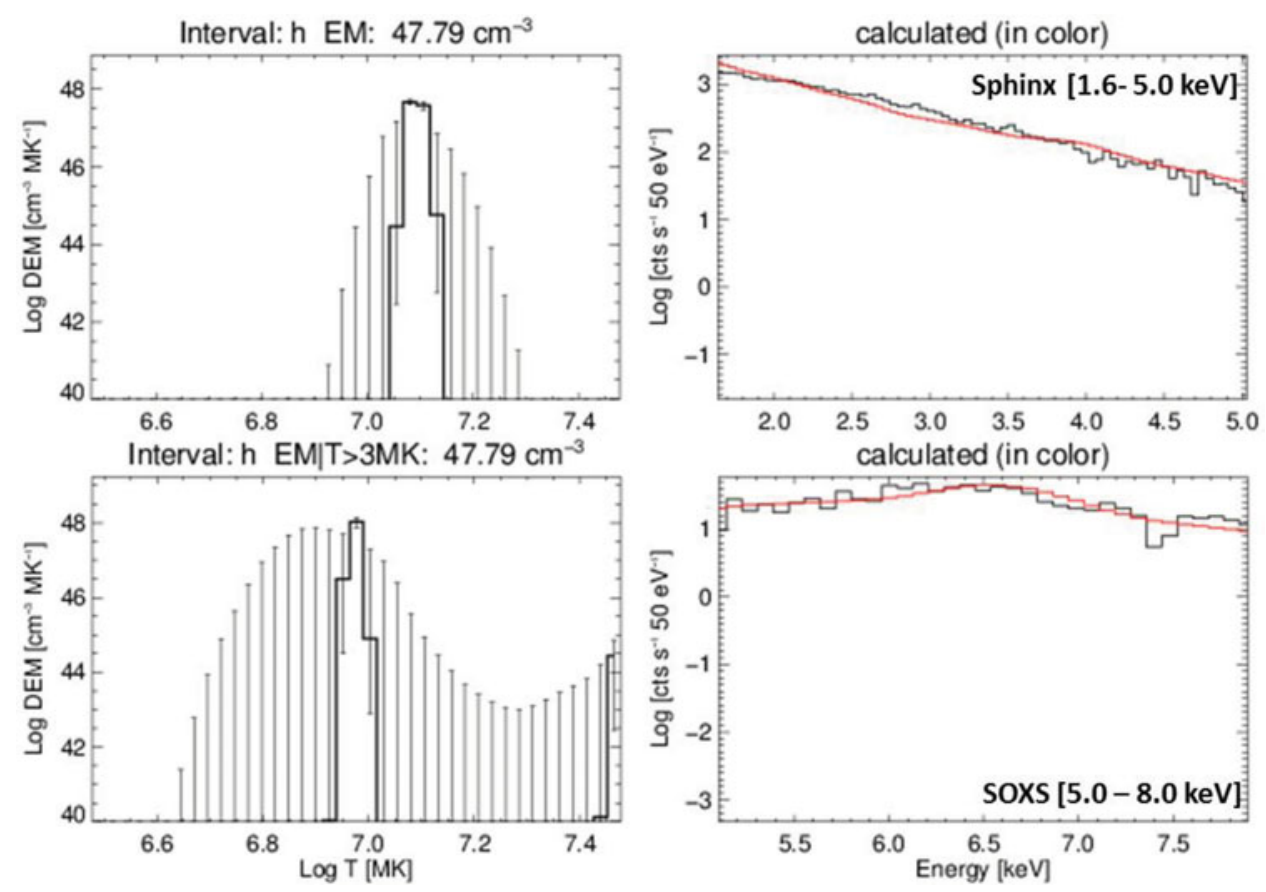

Figure 2. Top row: Best-fit $D E M-T$ relationship as well as the spectral-fit (drawn by red color) employing W-S inversion algorithm for the emission in 1.6-5.0 keV (plotted by black color), recorded by SphinX during 04:36:00-04:38:30 UT. Bottom row: Best-fit DEM-T and fitted SOXS spectrum in $5.0-8.0 \mathrm{keV}$ for the aforesaid time duration.

difference with the input observed spectrum (Kepa et al. (2008)). Coronal abundances are adopted from CHIANTI atomic database (Del Zanna et al. (2015)) while deriving the shape of theoretical spectra. As an input to this method, we have used fluxes recorded in the 73 energy bins (corresponding to the energy band 1.6-5.0 keV) and 35 energy bins (corresponding to the energy band 5.0-8.0 keV) by SphinX and SOXS instruments, respectively. The X-ray spectra are analyzed for various time duration as shown by dotted lines in the left panel of Fig. 1. Top row of the Fig. 2 shows the best-fit $D E M-T$ relation derived by analyzing X-ray spectrum in 1.6-5.0 keV (low-energy), observed by SphinX during the peak of the impulsive phase of the flare, 04:36:00-04:38:30 UT. Similarly, in the bottom panel, we present the best-fit $D E M-T$ curve and spectral-fit over the X-ray spectrum in 5.0-8.0 keV (high-energy), observed by SOXS during the aforesaid time.

From Fig. 2, it may be noted that the best-fit $D E M-T$ relation derived from SphinX observation suggests nearly isothermal nature of the $D E M$, with the peak at temperature $\left(T_{p}\right) \sim 13 \mathrm{MK}$. Similarly, the best-fit $D E M$ to the SOXS spectrum in 5.0-8.0 keV energy band for the same time interval suggests isothermal nature in the form of single gaussian function dependence on $T$, however, at $T_{p}=9.8 \mathrm{MK}$. Next, thermal energy are derived from the best-fit $D E M-T$ curve of Sphinx and SOXS observations and estimated to be 5.1 and $3.6 \times 10^{29} \mathrm{ergs}$, respectively. We employ the volume estimated from EIT/SOHO EUV wavelength images as shown in Fig. 1 for the calculation of thermal energies.

\section{Summary and Conclusions}

We study the thermal characteristics of the plasma during SOL2009-07-04T04:37 (B8.3) flare by analyzing its X-ray spectrum in various energy bands, as obtained by 
SphinX and SOXS instruments. We summarize the preliminary findings of this study as follows:

(a) Emission-measure is found to be of isothermal nature during the peak of the impulsive phase of the flare.

(b) Thermal energy and the temperature estimated by analyzing low-energy (from SphinX) and high-energy (from SOXS) bands within SXR spectrum result in different peak temperature as well as thermal energy.

In the next step, we have made a detailed investigation of thermal characteristics as well as the evolution of $D E M$ - $T$ relationship in various phases of the flare by combining the observations from SphinX and SOXS instruments. The corresponding paper is under review in the ApJ Main Journal (Awasthi et al. (2016)).

\section{Acknowledgements}

This research has been supported by Polish grant UMO-2011/01 /M/ST9/06096. Moreover, the research leading to these results has received funding from the European Community's Seventh Framework Programme (FP7/2007-2013) under grant agreement no. 606862 (F-CHROMA).

\section{References}

Awasthi, A. K., Sylwester, B., Sylwester, J., \& Jain, R. 2016, Submitted to the ApJ

Brown, J. C. 1971, Solar Phys., 18, 489.

Craig, I. J. D. \& Brown, J. C. 1976, A\& A, 49, 239.

Del Zanna, G., Dere, K. P., Young, P. R., Landi, E., \& Mason, H. E. 2015, A\& A, 582, A56

Gburek, S., Sylwester, J., Kowalinski, M., Bakala, J., Kordylewski, Z., Podgorski, P., Plocieniak, S., Siarkowski, M., Sylwester, B., Trzebinski, W., Kuzin, S. V., Pertsov, A. A., Kotov, Y. D., Farnik, F., Reale, F., \& Phillips, K. J. H. 2013, Solar Phys., 283, 631.

Jain, R., Awasthi, A. K., Rajpurohit, A. S., \& Aschwanden, M. J. 2011, Solar Phys., 270, 137. Jain, R., Dave, H., Shah, A. B., Vadher, N. M., Shah, V. M., Ubale, G. P., Manian, K. S. B., Solanki, C. M., Shah, K. J., Kumar, S., Kayasth, S. L., Patel, V. D., Trivedi, J. J., \& Deshpande, M. R. 2005, Solar Phys., 227, 89.

Kepa, A., Sylwester, B., Siarkowski, M., \& Sylwester, J. 2008, Adv. Sp. Res., 42, 828.

Sylwester, J., Schrijver, J., \& Mewe, R. 1980, Solar Phys., 67, 285 